\title{
Molecular beam epitaxy of CuMnAs
}

Krizek, Filip; Kašpar, Zdenk; Vetushka, Aliaksei; Kriegner, Dominik; Fiordaliso, Elisabetta M.; Michalicka, Jan; Man, Ondej; Zubá, Jan; Brajer, Martin; Hills, Victoria A.

Total number of authors:

16

Published in:

Physical Review Materials

Link to article, DOI:

10.1103/PhysRevMaterials.4.014409

Publication date:

2020

Document Version

Publisher's PDF, also known as Version of record

Link back to DTU Orbit

Citation (APA):

Krizek, F., Kašpar, Z., Vetushka, A., Kriegner, D., Fiordaliso, E. M., Michalicka, J., Man, O., Zubá, J., Brajer, M., Hills, V. A., Edmonds, K. W., Wadley, P., Campion, R. P., Olejník, K., Jungwirth, T., \& Novák, V. (2020).

Molecular beam epitaxy of CuMnAs. Physical Review Materials, 4(1), [014409].

https://doi.org/10.1103/PhysRevMaterials.4.014409

\section{General rights}

Copyright and moral rights for the publications made accessible in the public portal are retained by the authors and/or other copyright owners and it is a condition of accessing publications that users recognise and abide by the legal requirements associated with these rights.

- Users may download and print one copy of any publication from the public portal for the purpose of private study or research.

- You may not further distribute the material or use it for any profit-making activity or commercial gain

- You may freely distribute the URL identifying the publication in the public portal 


\title{
Molecular beam epitaxy of CuMnAs
}

\author{
Filip Krizek $\odot,{ }^{1, *}$ Zdeněk Kašpar $\odot,{ }^{1,2, *}$ Aliaksei Vetushka, ${ }^{1}$ Dominik Kriegner $\odot,{ }^{1,2}$ Elisabetta M. Fiordaliso, ${ }^{3}$ \\ Jan Michalicka, ${ }^{4}$ Ondřej Man $\odot,{ }^{4}$ Jan Zubáč $\odot,{ }^{1,2}$ Martin Brajer $\odot,{ }^{1,2}$ Victoria A. Hills, ${ }^{5}$ Kevin W. Edmonds, ${ }^{5}$ \\ Peter Wadley, ${ }^{5}$ Richard P. Campion ${ }^{5}{ }^{5}$ Kamil Olejník, ${ }^{1}$ Tomáš Jungwirth, ${ }^{1,5}$ and Vít Novák ${ }^{1, \dagger}$ \\ ${ }^{1}$ Institute of Physics, Czech Academy of Sciences, Cukrovarnická 10, 16200 Praha 6, Czech Republic \\ ${ }^{2}$ Faculty of Mathematics and Physics, Charles University, Ke Karlovu 3, 121 16 Prague 2, Czech Republic \\ ${ }^{3}$ DTU Nanolab, Technical University of Denmark, Fysikvej, Building 307, DK-2800 Kgs. Lyngby, Denmark \\ ${ }^{4}$ Central European Institute of Technology, Brno University of Technology, Purkyňova 123, 612 00, Brno, Czech Republic \\ ${ }^{5}$ School of Physics and Astronomy, University of Nottingham, Nottingham NG7 2RD, United Kingdom
}

(Received 5 November 2019; published 31 January 2020)

\begin{abstract}
We present a detailed study of the growth of the tetragonal polymorph of antiferromagnetic CuMnAs by the molecular beam epitaxy technique. We explore the parameter space of growth conditions and their effect on the microstructural and transport properties of the material. We identify its typical structural defects and compare the properties of epitaxial CuMnAs layers grown on GaP, GaAs, and Si substrates. Finally, we investigate the correlation between the crystalline quality of CuMnAs and its performance in terms of electrically induced resistance switching.
\end{abstract}

DOI: 10.1103/PhysRevMaterials.4.014409

\section{INTRODUCTION}

The tetragonal phase of the antiferromagnetic CuMnAs [1] attracted significant attention due to the possibility to control its Néel vector orientation by electrical current. It was predicted for a family of collinear antiferromagnets with noncentrosymmetric spin sublattices that a uniform electrical current induces an effective magnetic field with sign alternating between the magnetic sublattices, resulting in reorientation of the magnetic moments [2]. So far, current-induced Néel vector switching has been experimentally demonstrated in two prototypical examples of this materials family: in $\mathrm{CuMnAs}$ [3] and $\mathrm{Mn}_{2} \mathrm{Au}$ [4]. Moreover, further investigation of CuMnAs devices has recently uncovered an additional mechanism of high-resistive analog switching [5] ascribed to antiferromagnetic domain fragmentation generated by electrical and optical pulses of lengths ranging from microseconds to femtoseconds. Combined with the earlier observations of current-induced Néel vector switching, this makes the material potentially suitable for developing memory, optoelectronic and neuromorphic devices. The complexity of the switching mechanisms in CuMnAs and the broad range of considered device concepts call for a comprehensive materials growth and structural characterization study which is presented in this paper.

A favorable property of CuMnAs is the high degree of structural compatibility to common semiconductor materials, specifically to $\mathrm{GaAs}, \mathrm{GaP}$, and $\mathrm{Si}$. This makes it possible to employ molecular beam epitaxy (MBE) as the growth technique and to take full advantage of its precise control over the growth parameters. In this work we focus on the

\footnotetext{
*These authors contributed equally to this work.

†novakvit@fzu.cz
}

investigation of the growth conditions and material characterization of thin films of CuMnAs. We provide results from atomic force microscopy (AFM), superconducting quantum interference device (SQUID) magnetometry, $\mathrm{x}$-ray diffraction (XRD), and transport measurement techniques, and use them to identify the optimal growth conditions of stoichiometric CuMnAs. Using scanning transmission electron microscopy (STEM) we also identify two dominant types of crystallographic defects. Finally, we relate the resistive switching performance of CuMnAs microdevices to the studied growth conditions and crystalline quality of the films.

\section{GROWTH ON GaP}

The crystal structure of tetragonal CuMnAs [1,6] is shown in Fig. 1(a). Antiferromagnetically ordered magnetic moments are arranged in two inversion-partner sublattices [7]. Successful epitaxy of CuMnAs is achievable on GaP, GaAs, and $\mathrm{Si}$ (001) substrates, with the CuMnAs thin film growing under $45^{\circ}$ in-plane rotation [1], i.e., the $\langle 100\rangle$ direction of $\mathrm{CuMnAs}$ aligns with the $\langle 110\rangle$ direction of the substrate. From the three substrate materials, GaP can be expected to yield results superior to both GaAs with large lattice mismatch and Si with nonpolar surface and 1/4 unit-cell surface steps.

Our typical sample structure is shown in Fig. 1(b). The buffer layer improves the quality of the GaP-CuMnAs interface and the $\mathrm{Al}$ capping layer serves as a protection against oxidation at ambient conditions. For our growth conditions (more details are given in Methods [8]), the $(2 \times 4) \mathrm{GaP}$ surface reconstruction transforms to the $(2 \times 2) \mathrm{CuMnAs}$ reconstruction within the first $1-2 \mathrm{~nm}$ and then remains stable during further growth. The transition is shown in Fig. 1(c) for the GaP buffer layer viewed along the [110] direction and $50 \mathrm{~nm}$ thick CuMnAs viewed along the same azimuth $(\langle 100\rangle$ direction of CuMnAs). After the transition period, 
(a)

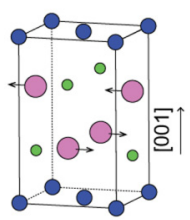

(c)

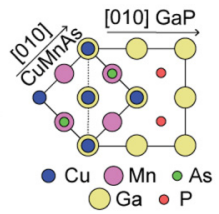

(b)
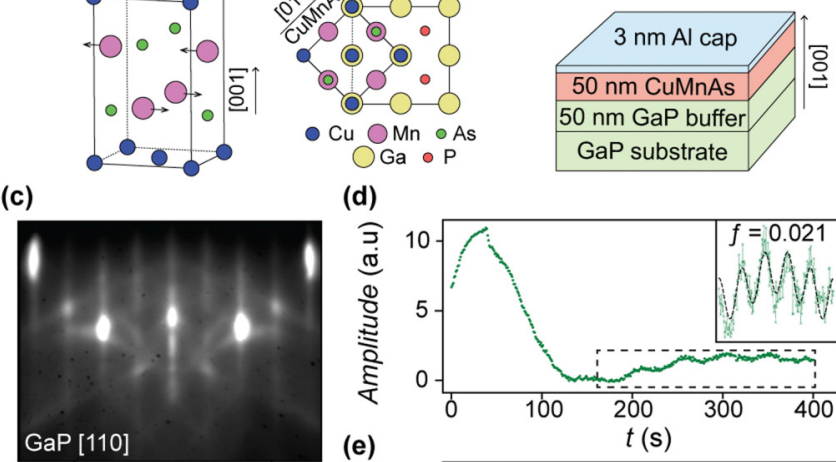

(d)
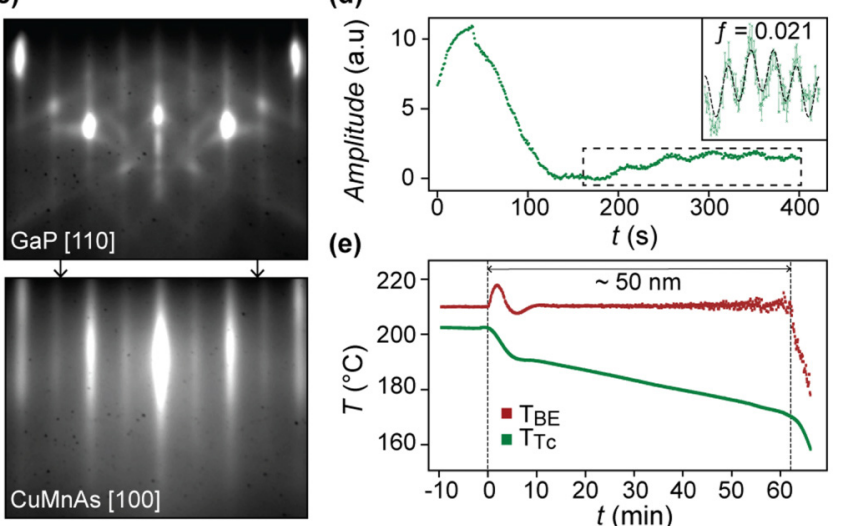

(e)

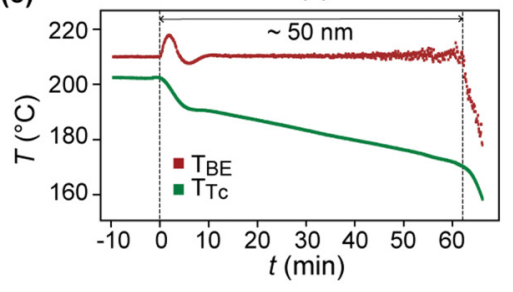

FIG. 1. (a) Model of tetragonal CuMnAs unit cell, where the black arrows show the expected orientation of magnetic moments (left panel). Model of CuMnAs matching the GaP unit cell, viewed from [001] direction (right panel). (b) Sketch of the cross-sectional structure of our standard samples. (c) Typical RHEED image of $2 \times$ reconstruction of (001) $\mathrm{GaP}$ buffer surface taken along the [110] crystal axis (top panel). Corresponding $2 \times$ reconstruction of CuMnAs taken under the same direction after $50 \mathrm{~nm}$ of growth (bottom panel). (d) RHEED oscillations acquired at the beginning of CuMnAs growth, with growth rate equal to 0.021 unit cell per second. (e) Time dependent evolution of growth temperature $T_{\mathrm{BE}}$ measured and regulated from the position of the optical absorbtion edge of the GaP substrate (red curve). Corresponding growth temperature $T_{T c}$ measured by a thermocouple at the substrate holder (green curve).

weak reflection high-energy electron diffraction (RHEED) oscillations appear, as shown in Fig. 1(d). The comparison of the layer thickness measured by x-ray reflectivity and/or atomically resolved STEM imaging shows that one RHEED oscillation period (here $\sim 47 \mathrm{~s}$ ) corresponds to the growth of one full CuMnAs unit cell, in contrast to nonmagnetic zinc-blende materials with one period per one monolayer.

A reliable substrate temperature readout is crucial for a reproducible CuMnAs growth. For this reason we measure the real-time optical absorption spectra of the substrate and extract the substrate temperature $T_{\mathrm{BE}}$ from the position of the absorption edge during growth $[9,10]$. The true substrate temperature measured this way significantly deviates from the usual thermocouple, whose reading $T_{T c}$ is delayed and more strongly linked to the substrate heater than to the substrate. If a real-time proportional-integral-derivative (PID) controller is used to maintain $T_{\mathrm{BE}}$ constant, both heater power and $T_{T c}$ decrease during growth with almost linear trend, as shown in Fig. 1(e). With our setup the absorption edge could be reliably resolved up to approximately $50 \mathrm{~nm}$ of growth. During longer growths, we extrapolate the linear decrease of the heater power to maintain a stable temperature. (a)

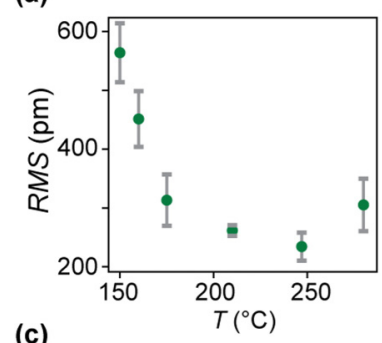

(b) $50 \mathrm{~nm}$

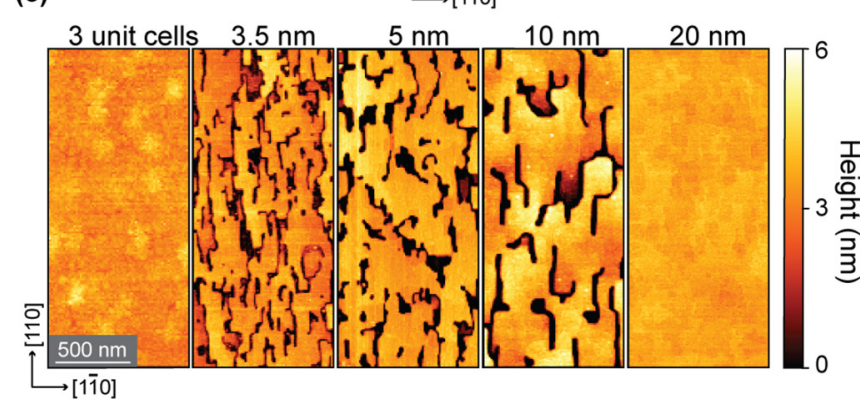

FIG. 2. (a) Dependency of surface roughness on growth temperature, for $50 \mathrm{~nm}$ thick CuMnAs samples grown on GaP substrate. (b) AFM micrograph of the surface of $50 \mathrm{~nm}$ thick CuMnAs grown at $T_{\mathrm{BE}}=210^{\circ} \mathrm{C}$. The height profile of a line cut through one of the terraces (green line) is shown in the inset. (c) AFM micrographs of CuMnAs layers grown on $\mathrm{GaP}$ with thickness varying from $1.9 \mathrm{~nm}$ (equivalent of 3 unit cells) to $20 \mathrm{~nm}$. The highlighted crystallographic directions correspond to the orientation of the substrate.

The importance of growth temperature is shown in Fig. 2(a) in terms of the surface roughness of $50 \mathrm{~nm}$ thick CuMnAs layers grown on GaP. The measured trend reveals that optimal growth temperatures are constrained to a window from 190 to $260^{\circ} \mathrm{C}$. The surface roughness measured for samples grown within this temperature window stems mainly from terraces shown in Fig. 2(b). The terraces are most frequently terminated by steps, which correspond to multiples of vertical lattice constant. This is in agreement with the unitcell periodicity of RHEED oscillations. Both observations illustrate the tendency of the CuMnAs crystal to grow in vertical steps containing two compensated monolayers of the magnetic element.

The AFM micrographs in Fig. 2(c) depict how the surface evolves for samples with varying CuMnAs thickness. The deposited material forms localized islands for the growth time equivalent to three unit cells. As the growth continues the islands coalesce into a percolation layer with a dense array of holes that are prevailingly elongated along the [110] direction of the GaP substrate. For even longer growth times, the density of the patterns gradually reduces until a continuous flat surface is formed. This growth regime is characteristic not only for GaP substrates, but we observe similar trends also on GaAs and $\mathrm{Si}$ as will be shown later in the text. The effect of the island formation in the initial stage of the growth is probably closely related to the formation of typical growth defects which will be analyzed and discussed later in the text. Under our typical growth conditions, it is possible to obtain flat and hole-free surface for thicknesses above $20 \mathrm{~nm}$. 
(a)

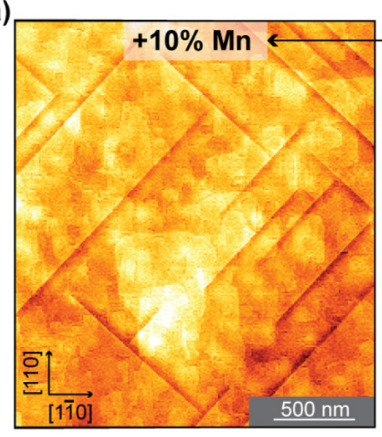

(c)

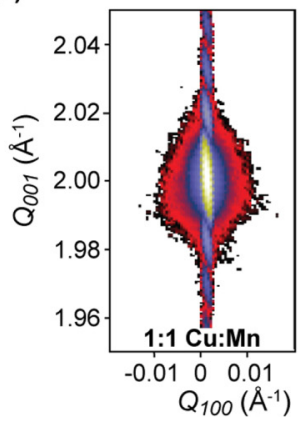

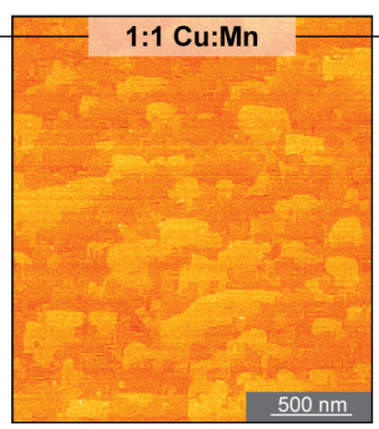

002 peak

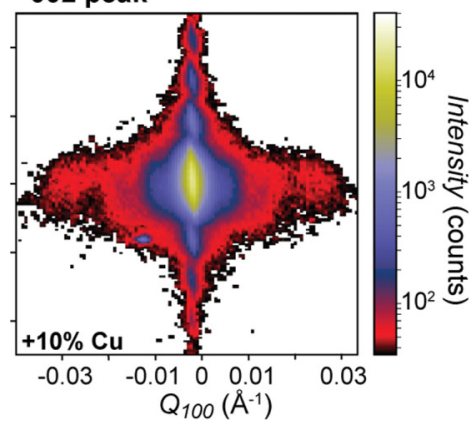

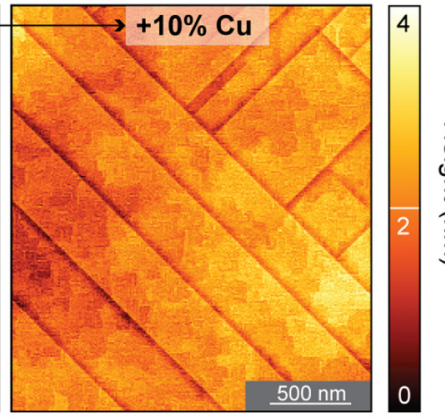

(d)

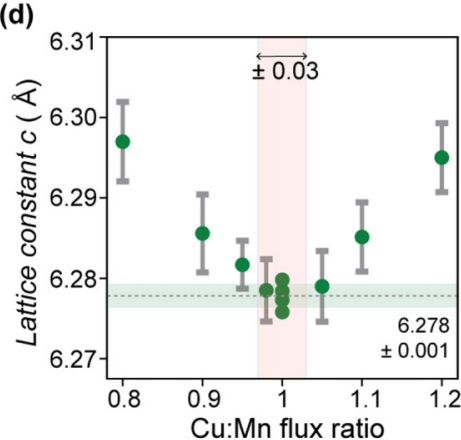

(b)

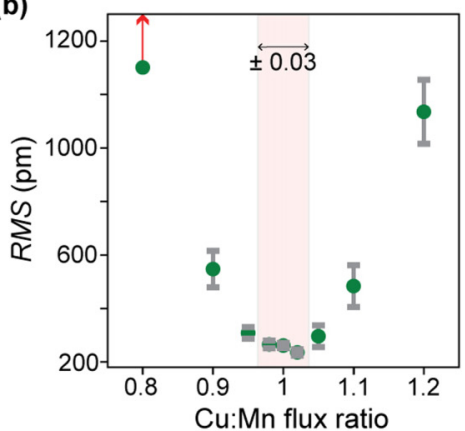

(e)

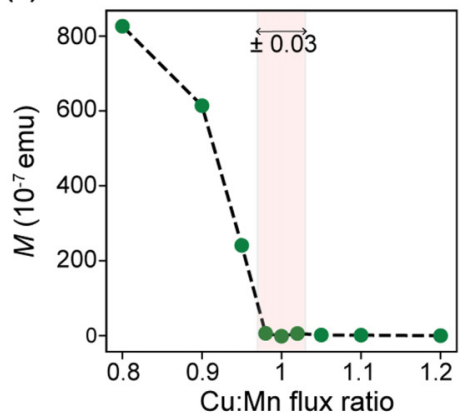

FIG. 3. (a) AFM micrograph of the surface of $50 \mathrm{~nm}$ thick Mn-rich, 1:1 stoichiometric, and Cu-rich CuMnAs samples grown on GaP. (b) Dependency of surface roughness of $50 \mathrm{~nm}$ thick CuMnAs layers on $\mathrm{Cu}: \mathrm{Mn}$ flux ratios. The highlighted area corresponds to the typical uncertainty of the beam flux measurements by the ion gauge. (c) Reciprocal space map of $002 \mathrm{CuMnAs}$ peaks for a 1:1 Cu:Mn sample (left panel) and a sample with excess copper (right panel). (d) Dependency of CuMnAs lattice constant $c$ on the Cu:Mn flux ratio. (e) Dependency of saturated magnetic moment $M$ on the $\mathrm{Cu}: \mathrm{Mn}$ flux ratio. The highlighted crystallographic directions correspond to the orientation of the substrate.

\section{STOICHIOMETRY}

Owing to low growth temperature, the epitaxial growth of CuMnAs does not require flux overpressure of any of the elements to compensate for an incongruent re-evaporation. However, arsenic incorporation turns out to be, to a certain extent, self-controlled. We have verified that the growing crystal tolerates As flux overpressure up to a factor of 2 (relative to $\mathrm{Cu}$ and $\mathrm{Mn}$ flux intensities) without any significant effect on the crystalline quality measured by, e.g., surface morphology, defect density, or electrical performance. On the other hand, these parameters sensitively depend on the ratio of the $\mathrm{Cu}$ and Mn fluxes. Given the limited accuracy of the independent flux calibrations and considering the unknown sticking coefficient of these elements, we have adopted the sharp minimum of the RMS surface roughness in a series of samples with varying $\mathrm{Cu}: \mathrm{Mn}$ flux ratio as a sign of the 1:1:1 stoichiometry. We have verified that the composition of the corresponding layer measured by the electron-probe microanalysis (EPMA) and energy-dispersive $\mathrm{x}$-ray spectroscopy (EDX) is correct within the $2 \%$ accuracy of the methods, and we will show further in the text that the optimal stoichiometry point is consistently confirmed by several other parameters.

A general trend in the surface morphology as a function of the $\mathrm{Cu}$ :Mn flux ratio is shown in Fig. 3(a). While the 1:1 $\mathrm{Cu}: \mathrm{Mn}$ sample has a flat surface with terraces of the unit-cell step height, the off-stoichiometric samples exhibit characteristic line-shaped surface defects oriented along the [110] and [110] directions of CuMnAs. As shown in Figs. 3(a) and 3(b), the density of these defects and so the RMS roughness steeply increase with the deviation from the 1:1 stoichiometry point. The defects are not related just to the surface of the layers, but they protrude through the bulk and leave a clear trace in the XRD reciprocal maps in form of a peak broadening along $Q_{100}$ axis, as shown in Fig. 3(c). The broadening gradually rises for off-stoichiometric samples up to the $\mathrm{Cu}: \mathrm{Mn}$ flux ratio of 1.1 and down to 0.9 . Outside these limits, the broadening becomes saturated or even slightly decreases which can be attributed to precipitation of the element in excess. Let us note that the RMS roughness in the outermost points of Fig. 3(b) has been measured in areas between these precipitates.

For 1:1 Cu:Mn samples grown on $\mathrm{GaP}$, we extract the vertical lattice constant $c=6.278 \pm 0.001 \AA$ in the fully tensilestrained lattice, i.e., fully pseudomorphic growth where the lattice constant $a_{\mathrm{CuMnAs}}$ matches $a_{\mathrm{GaP}} / \sqrt{2}$. This value increases when the flux ratio deviates from the stoichiometry point and the lattice relaxation sets in, as shown in Fig. 3(d). The lattice relaxation is likely related to the formation of microtwin defects, associated with surface defect lines, and/or slip dislocations which will be discussed in the next section. In addition, low energy formation of $\mathrm{Mn} / \mathrm{Cu}$ vacancies and/or $\mathrm{Cu}-\mathrm{Mn}$ substitutions could be significant in the off-stoichiometric samples [11]. In samples with more than $10 \%$ off-stoichiometry the excess material starts forming precipitates which protrude to the surface of the samples. In case of excess $\mathrm{Mn}$, these precipitates, most probably in form of MnAs, generate measurable net magnetization. This is apparent from the results of SQUID magnetometry shown in Fig. 3(e), with more details given in the Supplemental Material [8]. The distance between the inclusions is typically 
(a)

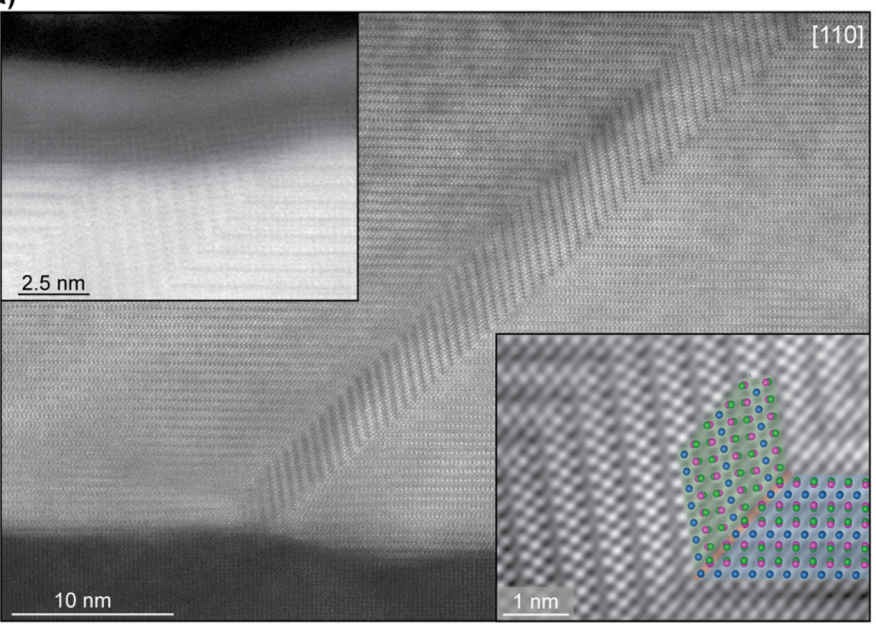

Cu $\mathrm{Mn} \odot \mathrm{As}$ (b)

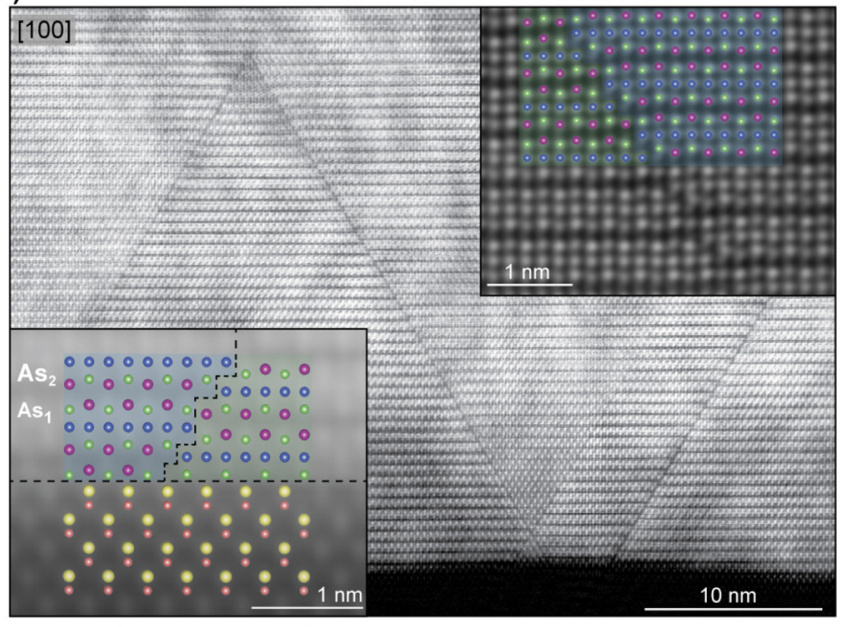

$\bigcirc \mathrm{Cu} \odot \mathrm{Mn} \odot \mathrm{As} \odot \mathrm{Ga} \odot \mathrm{P}$

FIG. 4. (a) HAADF-STEM micrograph of a twinlike structural defect propagating throughout $50 \mathrm{~nm}$ thick layer of CuMnAs and viewed from [110] CuMnAs (i.e., [100] GaP) direction. The lower inset shows a zoom in on the atomic structure of the defect, overallied with expected positions of $\mathrm{Cu}$-blue, $\mathrm{Mn}$ - purple, and As-green. The upper inset then shows the detail of the top interface with the Al cap. (b) HAADF-STEM micrograph of a slip dislocations presents the same $50 \mathrm{~nm}$ thick layer of CuMnAs, but viewed from [100] CuMnAs (i.e., [110] GaP) direction. The lower inset shows an atomic model overlay at the interface with the substrate (Ga-yellow, $\mathrm{P}-\mathrm{orange})$, where the first layer from the GaP substrate starts with either $\mathrm{Mn} / \mathrm{As}$ layer $\mathrm{As}_{1}$ or $\mathrm{As}_{2}$. The upper inset shows zoom in on the atomic structure of one of the defects, with the atomic model overlay representing the expected structure.

on the order of units to tens of $\mu \mathrm{m}$, which indicates surprisingly long diffusion length of excess $\mathrm{Cu}$ and $\mathrm{Mn}$ at given growth temperatures. These results are supported by magnetic force microscopy measurements shown in the Supplemental Material [8].

\section{STRUCTURAL DEFECTS}

We have prepared thin lamelae from various $50 \mathrm{~nm}$ thick CuMnAs samples by focused ion beam (FIB) technique in order to study the atomic structure of the defects. The high-angle annular dark field-scanning transmission electron microscopy (HAADF-STEM) analysis revealed two characteristic types of defects which were present in all studied samples. The first type is visible in the [110] projection of CuMnAs, as shown in Fig. 4(a). It is of the microtwin type and consists of a thin slab of CuMnAs crystal lattice rotated by 81.9 $\mathrm{deg}$, which corresponds to the angle between (111) and ( $\overline{1} \overline{1} 1)$ planes. The slab propagates through the whole thickness of the $50 \mathrm{~nm}$ CuMnAs film, mostly following the same (111) or (1̄11) planes. At the surface the microtwin slabs project as characteristic defect lines along [100] and [010] directions of CuMnAs, as shown in Fig. 3(a). Based on the depth profile measured across the surface defect lines by AFM, it is possible to determine the sign of the tilt of the corresponding microtwin beneath (see the upper inset in Fig. 4(a) and the Supplemental Material [8]). The typical width of the microtwins is several nanometers. As was shown in Fig. 3(b), there is a strong diffraction peak broadening and its magnitude scales with the microtwin density. It is clear that it does not directly reflect the crystalline structure of the defects. This is because the lattice spacing along the [001] direction of the twin has a different value after the rotation and therefore does not project into the $002 \mathrm{CuMnAs}$ reciprocal peaks. Yet, the STEM images in the Supplemental Material [8] show that there is a significant amount of strain in the CuMnAs crystal surrounding the microtwins. Therefore, the microtwin density is directly correlated to the amount of strain in the CuMnAs crystal, which projects as the winglike diffraction broadening of the reciprocal peaks. Finally, it should be noted that after investigating multiple different samples, we have not found any consistent correlation between the microtwin defect location and the local morphology of the underlying substrate (presence of surface steps, misfit dislocations, or other types of defects). The only direct correlation we have found is that the defect density increases with the deviation from the 1:1 stoichiometry point.

The second type of the characteristic defects can be seen on lamelae cut along the (100) planes of CuMnAs. Their typical structure is shown in Fig. 4(b). The apparent c/2 slip dislocations form antiphase boundaries along the (011) or (011) planes and always start at the substrate-film interface, similarly to the microtwin defects. They can either protrude throughout the whole thickness of the film or annihilate in a finite depth when two such defects with opposite tilt meet. It is important to note that only a fraction of the antiphase boundaries originate at the lattice steps on the substrate, which is otherwise typical for this type of defect [12]. Here, an additional mechanism for antiphase boundary formation on an atomically flat surface of the substrate is illustrated in the inset of Fig. 4(b). While the group-V sublattice of the substrate remains equally retained in the CuMnAs film, the tetragonal CuMnAs lattice may start either with the lower As plane $\left(\mathrm{As}_{1}\right)$ or with the upper As plane $\left(\mathrm{As}_{2}\right)$ rotated by $90^{\circ}$. This corresponds to a change in the stacking of the $\mathrm{Mn}$ and $\mathrm{Cu}$ layers in the individual grains. As a result, the $\mathrm{c} / 2$ lattice shift antiphase boundaries form when islands with different stacking come into contact during further growth. 
This type of defect may also be related to the formation of the holes present in the thin CuMnAs films. As apparent from Fig. 2(c), there is a transition from the growth of individual islands into a percolation layer with holes elongated along the [110] direction of the substrate for films with thickness between three unit cells and $3.5 \mathrm{~nm}$. These holes can then be observed in films with thicknesses up to $\sim 20 \mathrm{~nm}$, by which point the vast majority have disappeared. Considering the faceted profile of the holes, a possible explanation for their creation could be that lateral growth on these facets is locally suppressed at certain points when several antiphase islands meet. That would lead to a formation of faceted holes which can remain stable until enough adatoms accumulate and promote overgrowth into a smooth film. The complexity of the hole shape would then be related to the merging of multiple grains at once, i.e., local formation of multiple incoherent boundaries in close proximity. The overgrowth into a homogeneous layer could be locally further suppressed by the presence of impurities or adatom clustering within the holes $[13,14]$. The hole elongation is consistent with the presence of a diffusion anisotropy on the polar III-V surface [15], yielding islands that are elongated in the [110] direction of the substrate prior to coalescence. Indeed, this trend can be observed in the far left-hand image of Fig. 2(c), where a long and probably anisotropic diffusion length is suggested by the large spacing, layout, and shape of the islands in films of thickness below the percolation threshold. The large spacing of the precipitates during off-stoichiometric growth, as mentioned above, is further evidence for a long diffusion length.

The antiphase boundary defects were present with similar density in all samples investigated by STEM. In essence, the antiphase boundaries appear at the bottom interface about every $30 \mathrm{~nm}$. Their density is lower in the upper part of the layer due to annihilation of antiphase boundaries with opposing tilt. We did not observe any dependency on the growth parameters within the established growth temperature window. Post growth heating experiments in the STEM have shown that the defect structure remains unchanged up to temperatures comparable to the growth temperature of optimized samples. Details are shown in the Supplemental Material [8].

\section{GROWTH ON DIFFERENT SUBSTRATES}

Epitaxial growth of CuMnAs is also possible on (001) $\mathrm{GaAs}$ and $\mathrm{Si}$ substrates and within a similar growth temperature window. However, the surface roughness is significantly higher for films grown on GaAs and $\mathrm{Si}$ substrates. This is shown in the AFM images in Fig. 5(a), where the color scales correspond to the difference between the lowest and highest feature of $3 \mathrm{~nm}$ for GaP, $16 \mathrm{~nm}$ for GaAs, and $27 \mathrm{~nm}$ for $\mathrm{Si}$. The vertical lattice constant, $c$, extracted from the symmetric XRD scan along (001) axis of a $50 \mathrm{~nm}$ thick film is $6.299 \pm 0.003 \AA$ for $\mathrm{GaAs}$ and $6.300 \pm 0.011 \AA$ for $\mathrm{Si}$; let us recall that $c=6.278 \pm 0.001 \AA$ in the strained layer grown on GaP.

XRD analysis of 105 and 002 reciprocal peaks shows that there is a significant amount of mosaic tilt present in CuMnAs grown on Si and GaAs, as shown in Fig. 5(b). The peak with mosaic tilt of $0.07^{\circ}$ sits shifted by $0.04 \%$ with respect to the
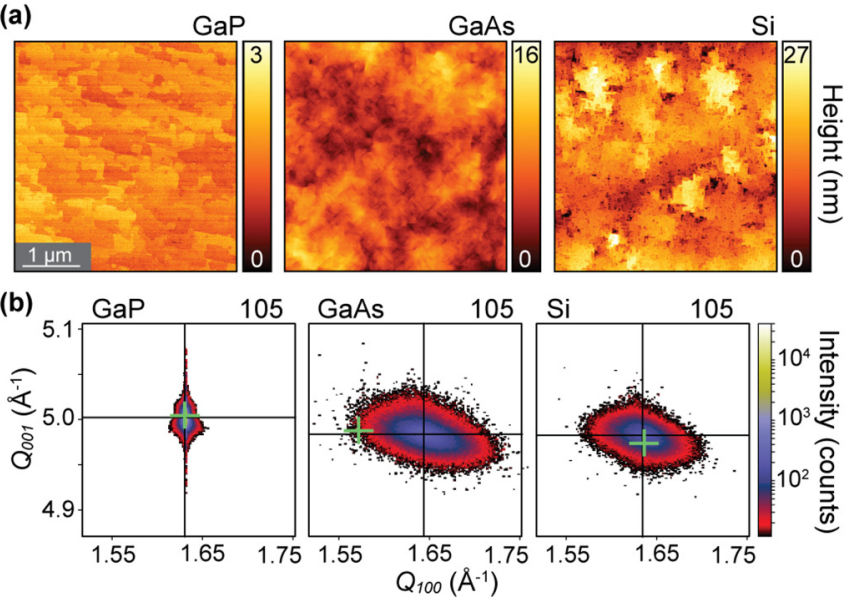

FIG. 5. (a) AFM scans of the surface of $50 \mathrm{~nm}$ thick CuMnAs layers grown on $\mathrm{GaP}, \mathrm{GaAs}$, and $\mathrm{Si}$ substrates, respectively. (b) Corresponding asymmetrical reciprocal space maps. The large black cross indicates the center of the peak and the green cross the expected peak center related to the position of 204 peak of the substrate.

position predicted from the reference 204 peak of the GaP substrate, which is a value we attribute to the fully strained case within the error of the measurement. The situation is similar for Si substrates with a shift of $0.10 \%$ and a mosaic tilt of $0.5^{\circ}$. As expected from the higher theoretical mismatch on GaAs, the peak is in this case shifted by $4.59 \%$ with a mosaic tilt of $1.0^{\circ}$. The corresponding lattice constants $a$ extracted from the asymmetrical peaks of the optimized samples are $3.853 \AA$ for GaP, $3.822 \AA$ for GaAs, and $3.844 \AA$ for Si. The mosaic tilt was converted into the lateral mosaic block size by an analytical model which is described in the Supplementl Material [8] and was adapted from Ref. [16]. The block size is the largest for CuMnAs on GaP, where it exceeds $400 \mathrm{~nm}$. For GaAs we extract a smaller block size of $\sim 40 \mathrm{~nm}$, as can be also expected due to the larger mismatch. Remarkably, the extracted block size for growth on $\mathrm{Si}$ is only $\sim 30 \mathrm{~nm}$, despite the low mismatch. This is because when CuMnAs is grown on As or $\mathrm{P}$ rich surface, it can coherently compensate $1 / 2$ of a lattice step on the substrate; $1 / 4$ steps are not probable due to the group $\mathrm{V}$ surface termination. This is different for $\mathrm{Si}$, where $1 / 4$ steps can be present. These steps cannot be coherently compensated and likely lead to the formation of defects which correlates with the increased mosaicity. In addition, a generally rougher $\mathrm{Si}$ surface can be expected due to the impossibility of growing Si buffer layers in our MBE system.

The CuMnAs/substrate interface was further investigated by STEM as shown in Fig. 6. In the case of GaP, the interface is pristine and the only disturbances are the microtwins and antiphase boundaries described above. This is different at the $\mathrm{CuMnAs} / \mathrm{GaAs}$ interface which is disturbed by arrays of misfit dislocations (with a slightly varying periodicity), as can be expected due to the large substrate/film mismatch. An example is shown in Fig. 6(b). The periodicity is around $\sim 26$ atomic columns and the CuMnAs layer is visibly strained around the interface. In contrast to GaP, the slip dislocations do not always protrude through the layer from the bottom 
(a)

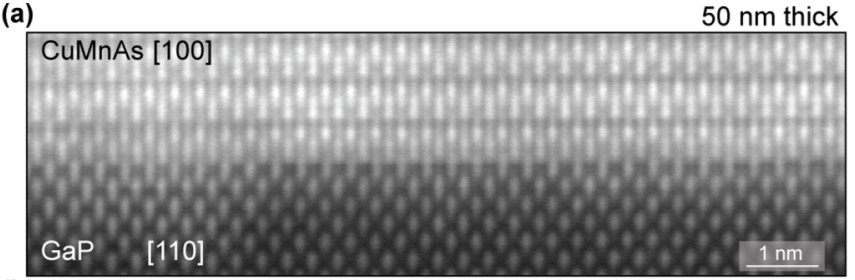

(b)

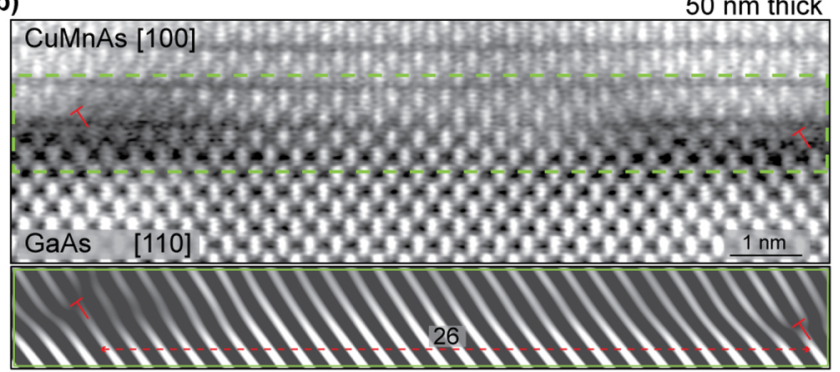

(c)

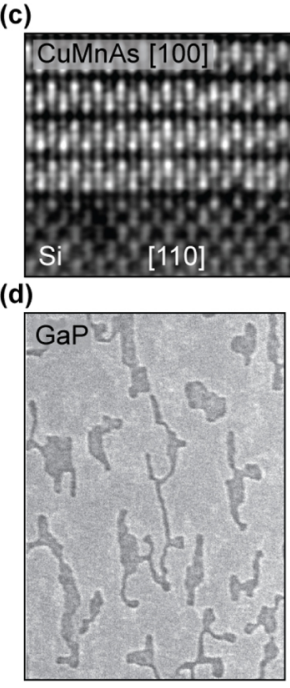

$50 \mathrm{~nm}$ thick
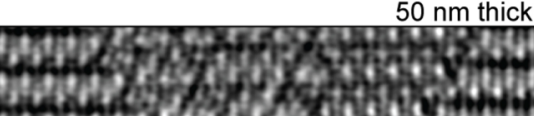

.

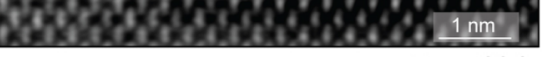

$5 \mathrm{~nm}$ thick
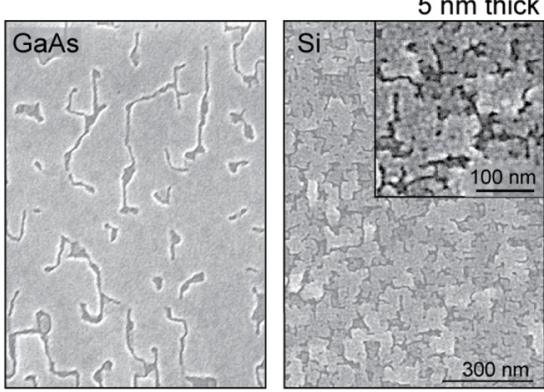

FIG. 6. (a) HAADF-STEM micrograph of the interface between CuMnAs and GaP, (b) GaAs with the area selected in green highlighting misfit dislocations (red markers) after filtering of the Fourier spectra and (c) Si. (d) SEM micrographs of $5 \mathrm{~nm}$ thick CuMnAs grown on $\mathrm{GaP}, \mathrm{GaAs}$, and Si substrates.

interface, but often terminate within the first few nanometers. This results in local straining of the layer and additional antiphase boundaries can appear within the strain field. Varying strain contrast and bending of the atomic rows is visible throughout the whole thickness of $50 \mathrm{~nm}$ samples.

The CuMnAs crystal is even more disturbed when grown on a Si substrate. An example of incoherently compensated surface step is shown in the bottom panel of Fig. 6(c). The step results in a formation of multiple crossing slip dislocations. In general, while growing on $\mathrm{Si}$, the crystal structure of CuMnAs films becomes very complex. The STEM analysis shows steps on the CuMnAs surface with height up to $30 \mathrm{~nm}$ for $50 \mathrm{~nm}$ thick layers, avalanche arrays of slip dislocations, and a variety of other crystallographic defects. These complex structures form above incoherent interfacial defects and disturb the film throughout the whole thickness. More STEM micrographs are shown in the Supplemental Material [8] for all $\mathrm{GaP}, \mathrm{GaAs}$, and Si substrates. (a)

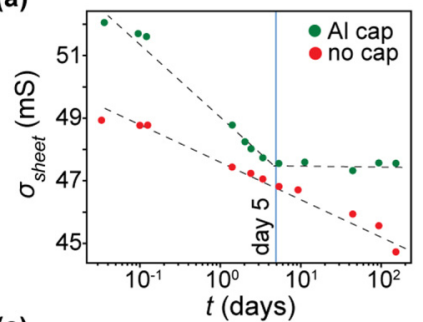

(c)

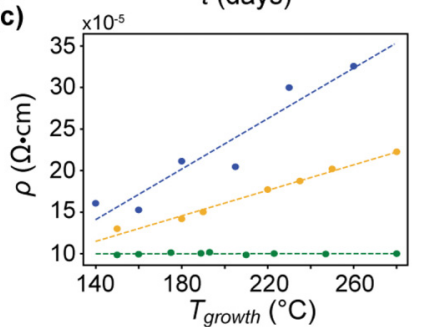

(b)

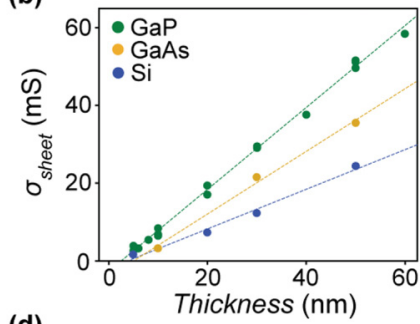

(d)

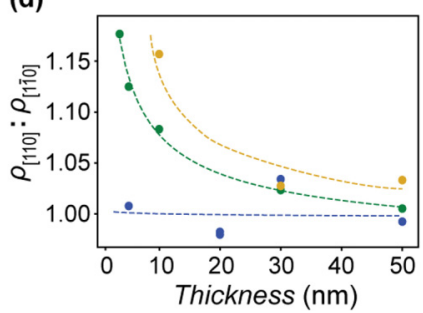

FIG. 7. (a) Evolution of sheet conductance $\sigma_{\text {sheet }}$ in time for $50 \mathrm{~nm}$ thick CuMnAs with $\mathrm{Al}$ cap (red) and without capping (green) grown on GaP substrate. (b) Dependency of $\sigma_{\text {sheet }}$ on the thickness of CuMnAs films grown on $\mathrm{GaP}, \mathrm{GaAs}$, and Si substrates. (c) Dependency of the resistivity $\rho$ on growth temperature for $50 \mathrm{~nm}$ thick films grown on $\mathrm{GaP}, \mathrm{GaAs}$, and Si substrates. (d) Ratio of resistivities measured along the [110] and [010] directions of the GaP substrate with varying thickness grown on $\mathrm{GaP}, \mathrm{GaAs}$, and Si substrates. The dashed lines are guides for the eye.

Despite the difference in lattice mismatch, CuMnAs films thinner than $20 \mathrm{~nm}$ tend to form layers with holes and/or isolated islands on all three substrates, as demonstrated in Fig. 6(d) for $5 \mathrm{~nm}$ thick layers. The mechanism of the formation of antiphase islands applies in all three cases. Only the island size is smaller and the density of holes significantly higher for the Si substrate. As already mentioned, this is likely due to the presence of the 1/4 lattice steps on the surface of $\mathrm{Si}$ and to its nonpolar surface. In contrast to films on GaAs and $\mathrm{GaP}$, there is no visible anisotropy and elongation of the holes in the film while grown on the nonpolar surface of Si. This observation can be linked to the (an)isotropy of the electrical resistance, as will be shown in the next section.

\section{ELECTRONIC PROPERTIES}

In general, unprotected CuMnAs films tend to degrade at ambient conditions due to surface oxidation, which affects the electrical properties. This is illustrated in Fig. 7(a), where the sheet conductance $\sigma_{\text {sheet }}$ and its time evolution are shown for samples of $50 \mathrm{~nm}$ thick CuMnAs film grown on $\mathrm{GaP}$, in one case unprotected and in the other case in situ capped with $3 \mathrm{~nm}$ of Al. At first, both conductivities rapidly decrease. The conductivity of the capped sample started at a higher value due to the additional conductance of the $\mathrm{Al}$ film. However, after approximately five days, $\sigma_{\text {sheet }}$ of the capped sample stabilizes when the $\mathrm{Al}$ cap oxidizes and forms a stable $\mathrm{AlO}_{x}$ layer. Oxidation of the unprotected layer continues with a rate that only logarithmically slows down over a period of over 150 days.

The conductance of CuMnAs films scales linearly with thickness, as shown in Fig. 7(b), except for ultrathin layers 

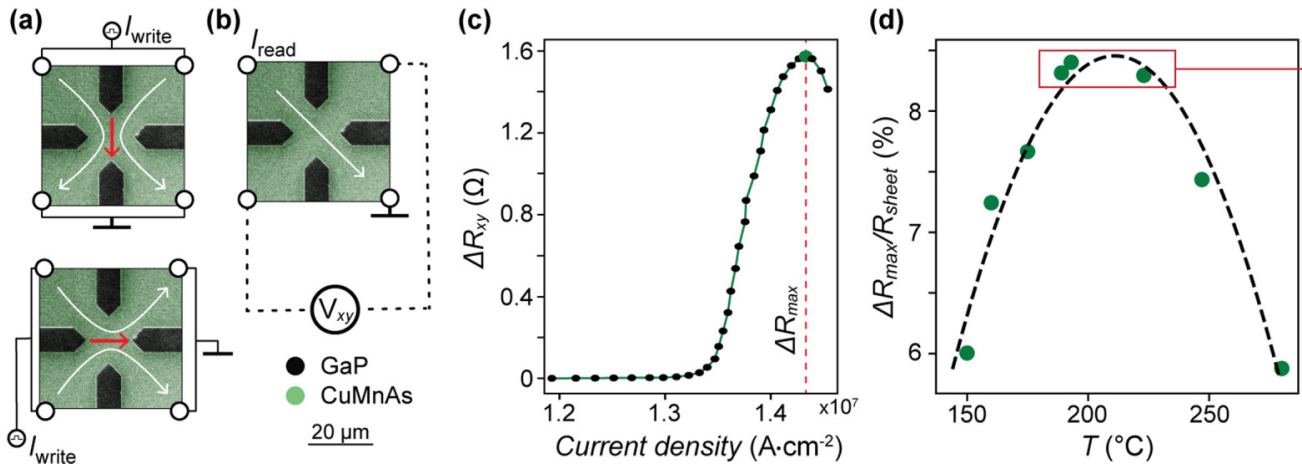

(e)

FIG. 8. (a) SEM micrographs of a typical cross device, where the white arrows indicate the current direction for the two orthogonal writing pulses in the top panels (net current directions are indicated by red arrow). (b) The same micrograph where the white arrow indicates the transversal resistance readout. (c) Typical dependency of transversal resistance $\Delta \mathrm{R}_{x y}$ on the pulse current density, with the maximum value $\Delta R_{\max }$ indicated by red dashed line. (d) Dependence of the maximal difference in normalized transversal resistance $\Delta R_{\max } / R_{\text {sheet }}$ on the growth temperature, with parabolic fit as guide for the eye. (e) The same dependence on Cu:Mn flux ratio. The highlighted point (red box) corresponds to the average value of samples grown within the temperature window between 180 and $220^{\circ} \mathrm{C}$ shown in (d).

below approximately $3 \mathrm{~nm}$, where percolation and/or quantum conductance effects step in. Apart from that, we see an onset of the conductance anisotropy for thicknesses below $20 \mathrm{~nm}$, with the conductance in the [110] direction of the GaP and GaAs substrates being systematically larger than that in the [110] direction, see Fig. 7(d). This is in line with the formation of the elongated islands and holes in thin films on GaP and GaAs, shown and discussed in previous sections. Let us note that no pronounced conductance anisotropy has been found in samples grown on $\mathrm{Si}$, again in accordance with the absence of substrate surface polarity. We note that the anisotropy of conductance is averaged out in the value measured in the Van der Pauw geometry.

The resistivity of stoichiometric CuMnAs films grown on GaP substrates is $\rho=1.0 \times 10^{-4} \Omega \mathrm{cm}$ and this value is virtually independent of the growth temperature in the range from 150 to $280^{\circ} \mathrm{C}$, see Fig. 7(c). Resistivities of CuMnAs grown on GaAs or $\mathrm{Si}$ decrease with decreasing growth temperature but remain higher compared to the on-GaP-grown material in the whole investigated range of temperatures. Below $140{ }^{\circ} \mathrm{C}$ we could perform only growth tests without the spectral temperature readout and the control of the growth temperature, resulting in a $3 \mathrm{D}$ growth and macroscopically rough surfaces.

The temperature dependence of the conductivity shows a clear metallic behavior on all substrates, see Supplemental Material [8]. The material grown on GaP showed the lowest residual resistivity at $4.2 \mathrm{~K}, 29 \%$ of the room temperature value. This corresponds to the superior crystal quality, compared to the layers grown on GaAs and $\mathrm{Si}$ substrates whose residual resistivities at $4.2 \mathrm{~K}$ are $56 \%$ and $85 \%$ of the room temperature values, respectively.

In order to correlate the investigated growth parameters to the material performance in terms of current-induced resistive switching $[3,5,17,18]$, simple cross structures were prepared by optical lithography and wet etching; details of the fabrication process are given in the Supplemental Material [8]. The experimental procedure and device geometry are similar to the previous work of Olejník et al. [18]. Pulses of $100 \mu \mathrm{s}$ duration and varying voltage were used for generating of writing currents with density on the order of $10^{7} \mathrm{~A} / \mathrm{cm}^{2}$. The pulses were applied in two perpendicular directions ([110] and [110] directions of GaP, Fig. 8(a)), at room temperature. The transversal resistance in a $45^{\circ}$ rotated direction [Fig. 8(b)] was measured $1 \mathrm{~s}$ after each of the writing pulses. The difference of the two transversal resistances, $\Delta \mathrm{R}_{x y}$, is plotted in Fig. 8(c) as a function of writing current density. Here, $\Delta \mathrm{R}_{x y}$ starts to rapidly grow after the pulse current exceeds a threshold value and reaches a maximum $\Delta R_{\max }$ that is specific for the given material. We take this maximum normalized to the sheet resistance of the sample, $\Delta R_{\max } / R_{\text {sheet }}$, as a material figure of merit in the switching experiments. Further increase in the pulsing voltage results not only in rapid decrease of $\Delta \mathrm{R}_{x y}$, but also in a permanent change in the resistivity of the device. This is likely because the heat induced by the pulse damages the CuMnAs crystal.

Let us note that the simple cross geometry is suitable for comparison with previous studies, since the measured signal contains both the weaker spin-orbit-torque induced anisotropic magnetoresistance contribution to the switching signal $[2,3,19,20]$ and the stronger increase in transversal voltage due to nonuniform change of longitudinal conductivity [5]. The latter contribution is dominant in sample geometries optimized for measurements of longitudinal conductivity, e.g., simple bars, Wheatstone bridge geometry, etc., and is reduced in the cross geometry. We find a conversion factor of 0.4 , while comparing measurements of $\Delta R_{\max } / R_{\text {sheet }}$ between the cross geometry and the bar geometry presented in Ref. [5]. The compared devices were fabricated from the same material.

The dependence of $\Delta R_{\max }$ on the growth temperature for the 1:1 Cu:Mn stoichiometric CuMnAs is shown in Fig. 8(d). There is a clear maximum of $\Delta \mathrm{R}_{\max } \sim 8.4 \%$ at growth temperatures between 190 and $230{ }^{\circ} \mathrm{C}$. This correlates with the minimum surface roughness that was shown in Fig. 2(a). Surprisingly, there is no similar trend in the plain resistivity of the material.

The dependence of $\Delta R_{\max }$ on the $\mathrm{Cu}: \mathrm{Mn}$ ratio is shown in Fig. 8(e). Again, we observe a maximum $\Delta R_{\max }$ of 
$8.34 \pm 0.08 \%$ for the three 1:1 Cu:Mn samples grown within the optimal temperature window. The value of $\Delta R_{\max }$ decreases when deviating from the 1:1 $\mathrm{Cu}: \mathrm{Mn}$ stoichiometric point. This implies that the presence of the microtwin defects does not foster the switching performance, as their density increases for off-stoichiometric samples. Moreover, the large achievable spacing between the microtwin defects in CuMnAs grown on $\mathrm{GaP}$ allows us to avoid them when fabricating devices. This is hardly possible with CuMnAs grown on GaAs and $\mathrm{Si}$, and the above measurements showed that $\Delta \mathrm{R}_{\max }$ is lower in these films, not exceeding $4.4 \%$ in case of GaAs and $4.0 \%$ in case of $\mathrm{Si}$.

\section{CONCLUSION}

We have demonstrated that epitaxy of tetragonal CuMnAs is possible on $\mathrm{GaP}, \mathrm{GaAs}$, and $\mathrm{Si}$ substrates. Exploring a range of growth parameters, we found a growth temperature and a material flux setting which result in a smooth surface morphology, the lowest defect density and best electrical switching performance of the CuMnAs films. We have shown that the trends of these three material properties coincide. Of the three substrates, GaP was found to result in the superior quality of the epitaxial layer.

We have identified two characteristic types of structural defects present in thin film CuMnAs. The microtwin defects clearly correlate with the growth parameters and leave a characteristic imprint on the surface of the films and also in the reciprocal space. The occurrence of these defects can be largely suppressed in layers grown on GaP. Density of the second type of defects, the antiphase boundaries, seems insensitive to fine adjustment of the growth conditions. This defect type is likely related to the 90-degree ambiguity in the orientation of nucleation islands of the epitaxial layer.

The observed defects may locally disturb the antiferromagnetic ordering in the material and provide potential pinning centers for magnetic domain formation [21,22]. A detailed theoretical study and/or further improvement of the crystal quality by implementing more complex growth protocols in order to grow antiphase boundary free material (e.g., seeding layers, migration enhancement, or growth temperature gradient) are necessary to unravel the effect which these defects may have on the performance of CuMnAs based spintronic devices.

\section{ACKNOWLEDGMENTS}

We thank J. Zyka, Z. Šobáň, V. Jurka, K. Hruška, and P. Zich for technical support. We also thank J.Gazquez, M. Roldan, and M. Varela from Oak Ridge National Laboratory for assistance with the STEM measurements. This work was supported in part by the Ministry of Education of the Czech Republic Grant Nos. LM2018096, LM2018110, and LNSM-LNSpin, the Grant Agency of the Czech Republic Grant No. 19-28375X, the Charles University Grant GA UK No. 886317, the EU FET Open RIA Grant No. 766566, the Engineering and Physical Sciences Research Council Grant No. EP/P019749/1). T.J. acknowledges the support from the Neuron Foundation Prize. K.O. acknowledges the support from the Neuron Foundation Impuls Grant. Part of the work was carried out with the support of CEITEC Nano Research Infrastructure (ID LM2015041, MEYS CR, 20162019), CEITEC Brno University of Technology.
[1] P. Wadley, V. Novák, R. Campion, C. Rinaldi, X. Martí, H. Reichlová, J. Železný, J. Gazquez, M. Roldan, M. Varela et al., Nat. Commun. 4, 2322 (2013).

[2] J. Železný, H. Gao, K. Výborný, J. Zemen, J. Mašek, A. Manchon, J. Wunderlich, J. Sinova, and T. Jungwirth, Phys. Rev. Lett. 113, 157201 (2014).

[3] P. Wadley, B. Howells, J. Železný, C. Andrews, V. Hills, R. P. Campion, V. Novák, K. Olejník, F. Maccherozzi, S. Dhesi et al., Science 351, 587 (2016).

[4] S. Y. Bodnar, L. Šmejkal, I. Turek, T. Jungwirth, O. Gomonay, J. Sinova, A. Sapozhnik, H.-J. Elmers, M. Kläui, and M. Jourdan, Nat. Commun. 9, 348 (2018).

[5] Z. Kašpar, M. Surýnek, J. Zubáč, F. Krizek, V. Novák, R. P. Campion, M. S. Wörnle, P. Gambardella, X. Marti, P. Němec et al., arXiv:1909.09071.

[6] K. Momma and F. Izumi, J. Appl. Crystallogr. 44, 1272 (2011).

[7] P. Wadley, V. Hills, M. Shahedkhah, K. Edmonds, R. Campion, V. Novák, B. Ouladdiaf, D. Khalyavin, S. Langridge, V. Saidl et al., Sci. Rep. 5, 17079 (2015).

[8] See Supplemental Material at http://link.aps.org/supplemental/ 10.1103/PhysRevMaterials.4.014409 for the following details: Methods; S1: Surface line defects and magnetic inclusions; S2: Identification of microtwin tilt in AFM; S3: Strain induced by the microtwin defects; S4: Atomic structure of CuMnAs grown on different substrates; S5: Effect of heating on CuMnAs; S6: Temperature dependence of resistance.

[9] V. Novák, K. Olejník, M. Cukr, L. Smrčka, Z. Remeš, and J. Oswald, J. Appl. Phys. 102, 083536 (2007).

[10] M. Weilmeier, K. Colbow, T. Tiedje, T. V. Buuren, and L. Xu, Can. J. Phys. 69, 422 (1991).

[11] F. Máca, J. Kudrnovský, P. Baláž, V. Drchal, K. Carva, and I. Turek, J. Magn. Magn. Mater. 474, 467 (2019).

[12] Z. Wang, H. Guo, S. Shao, M. Saghayezhian, J. Li, R. Fittipaldi, A. Vecchione, P. Siwakoti, Y. Zhu, J. Zhang et al., Proc. Natl. Acad. Sci. USA 115, 9485 (2018).

[13] K. Fujiwara, K. Kanamoto, Y. Ohta, Y. Tokuda, and T. Nakayama, J. Cryst. Growth 80, 104 (1987).

[14] H. Ye, L. Li, R. T. Hinkey, R. Q. Yang, T. D. Mishima, J. C. Keay, M. B. Santos, and M. B. Johnson, J. Vac. Sci. Technol. B 31, 03C135 (2013).

[15] K. Ohta, T. Kojima, and T. Nakagawa, J. Cryst. Growth 95, 71 (1989).

[16] V. Holy, T. Baumbach, and U. Pietsch, High-Resolution X-ray Scattering from Thin Films and Multilayers (Springer, Berlin, 1999).

[17] K. Olejník, V. Schuler, X. Martí, V. Novák, Z. Kašpar, P. Wadley, R. P. Campion, K. W. Edmonds, B. L. Gallagher, J. Garcés et al., Nat. Commun. 8, 15434 (2017). 
[18] K. Olejník, T. Seifert, Z. Kašpar, V. Novák, P. Wadley, R. P. Campion, M. Baumgartner, P. Gambardella, P. Němec, J. Wunderlich et al., Sci. Adv. 4, eaar3566 (2018).

[19] P. Wadley, S. Reimers, M. J. Grzybowski, C. Andrews, M. Wang, J. S. Chauhan, B. L. Gallagher, R. P. Campion, K. W. Edmonds, S. S. Dhesi et al., Nat. Nanotechnol. 13, 362 (2018).
[20] M. J. Grzybowski, P. Wadley, K. W. Edmonds, R. Beardsley, V. Hills, R. P. Campion, B. L. Gallagher, J. S. Chauhan, V. Novak, T. Jungwirth, F. Maccherozzi, and S. S. Dhesi, Phys. Rev. Lett. 118, 057701 (2017).

[21] T. Jourdan, F. Lançon, and A. Marty, Phys. Rev. B 75, 094422 (2007).

[22] A. Parthasarathy and S. Rakheja, Phys. Rev. Appl. 11, 034051 (2019). 